\title{
Исследование структурных и морфологических свойств НРНТ алмазных подложек
}

\author{
() П.А. Юнин ${ }^{1}$, П.В. Волков ${ }^{1}$, Ю.Н. Дроздов ${ }^{1}$, А.В. Колядин ${ }^{3}$, С.А. Королев ${ }^{1}$, \\ Д.Б. Радищев ${ }^{2}$, Е.А. Суровегина ${ }^{1}$, В.И. Шашкин ${ }^{1}$ \\ ${ }^{1}$ Институт фризики микроструктур Российской академии наук, \\ 603087 Нижний Новгород, Россия \\ ${ }^{2}$ Институт прикладной физики Российской академии наук, \\ 603950 Нижний Новгород, Россия \\ ${ }^{3}$ ООО „Нью Даймонд Технолоджи“, \\ 197706 Санкт-Петербург, Россия \\ E-mail: yunin@ipmras.ru
}

(Получена 25 апреля 2018 г. Принята к печати 7 мая 2018 г.)

\begin{abstract}
Методами оптической интерференционной микроскопии белого света, атомно-силовой микроскопии и рентгеновской дифрактометрии проведены комплексные исследования морфологических и структурных свойств серии монокристаллических НРНТ алмазных подложек. Описаны методики, позволяющие охарактеризовать наиболее критичные для эпитаксиального применения параметры подложек на лабораторном оборудовании. Показано, что характеризации алмазных подложек только по ювелирному типу недостаточно при оценке возможности их использования для эпитаксиального роста CVD-алмаза.
\end{abstract}

DOI: 10.21883/FTP.2018.11.46592.14

\section{1. Введение}

Известно, что монокристаллические алмазные подложки для гомоэпитаксиального роста CVD-алмаза (chemical vapor deposition) электронного качества производятся методом НРНТ (high pressure high temperature). В настоящее время НРНТ подложки имеют относительно малые размеры, высокое содержание остаточных примесей и неоднородность свойств по площади [1-6]. Высококачественная по оценке производителя подложка может не удовлетворять требованиям для роста эпитаксиальных структур электронного качества. Необходимо иметь методики контроля наиболее критичных для эпитаксии параметров подложек, таких как морфология поверхности и структурные свойства монокристалла. В литературе имеется множество примеров исследования структурных свойств НРНТ алмазов с использованием рентгеновских синхротронных источников излучения [1-3]. Однако такие методики из-за их сложности не пригодны для рутинного контроля технологии изготовления подложек. Топографические рентгеновские методы, несмотря на некоторые преимущества, также имеют свои ограничения по применимости и информативности количественного анализа [4,5].

Реализация методик диагностики критических структурных и морфологических свойств НРНТ монокристаллов на коммерческом лабораторном оборудовании позволит осуществлять рутинный контроль подложек, отбирая подложки с заданными свойствами для дальнейшего контролируемого роста и травления гомоэпитаксиальных структур [6] или же изготовления мозаичных подложек большой площади [7]. Поэтому знание этого параметра необходимо для получения гомоэпитаксиальных CVD слоев алмаза с заданной толщиной. Таким образом, разработка методик входного анализа, позволяющих оценить наиболее критические для эпитаксии характеристики подложки, в настоящее время чрезвычайно актуальна.

\section{2. Эксперимент}

В рамках работы были исследованы 6 образцов коммерческих НРНТ подложек производства ООО „New Diamond Technology“ (г. Санкт-Петербург, Россия), ювелирный тип II $a$, ориентация (001). Информация об углах среза подложек отсутствовала. Исследованные подложки имели латеральные размеры $4 \times 4$ и $3.5 \times 3.5$ мм. Из них три подложки были „моносекториальные“ - каждая была вырезана из одного сектора роста грани (001) отдельного НРНТ кристалла. Номинальная толщина подложек была указана 0.4-0.5 мм.

Методом низкокогерентной оптической интерферометрии проводилось картирование толщины подложек. Используемый метод измерения толщины прозрачного объекта основан на том, что для пучков частично когерентного света интерференция возникает только тогда, когда оптическая разность хода между этими пучками не превышает определенную величину, так называемую длину когерентности источника излучения. Соответственно, если свет от широкополосного источника последовательно проходит через две линии задержки (два интерфемерометра), то при совпадении величин задержек возникает интерференционный сигнал. При этом опорный интерферометр имеет возможность контролируемой перестройки разности длин плеч, а в качестве второго интерферометра выступает образец. Оптическая связь между опорным интерферометром и 

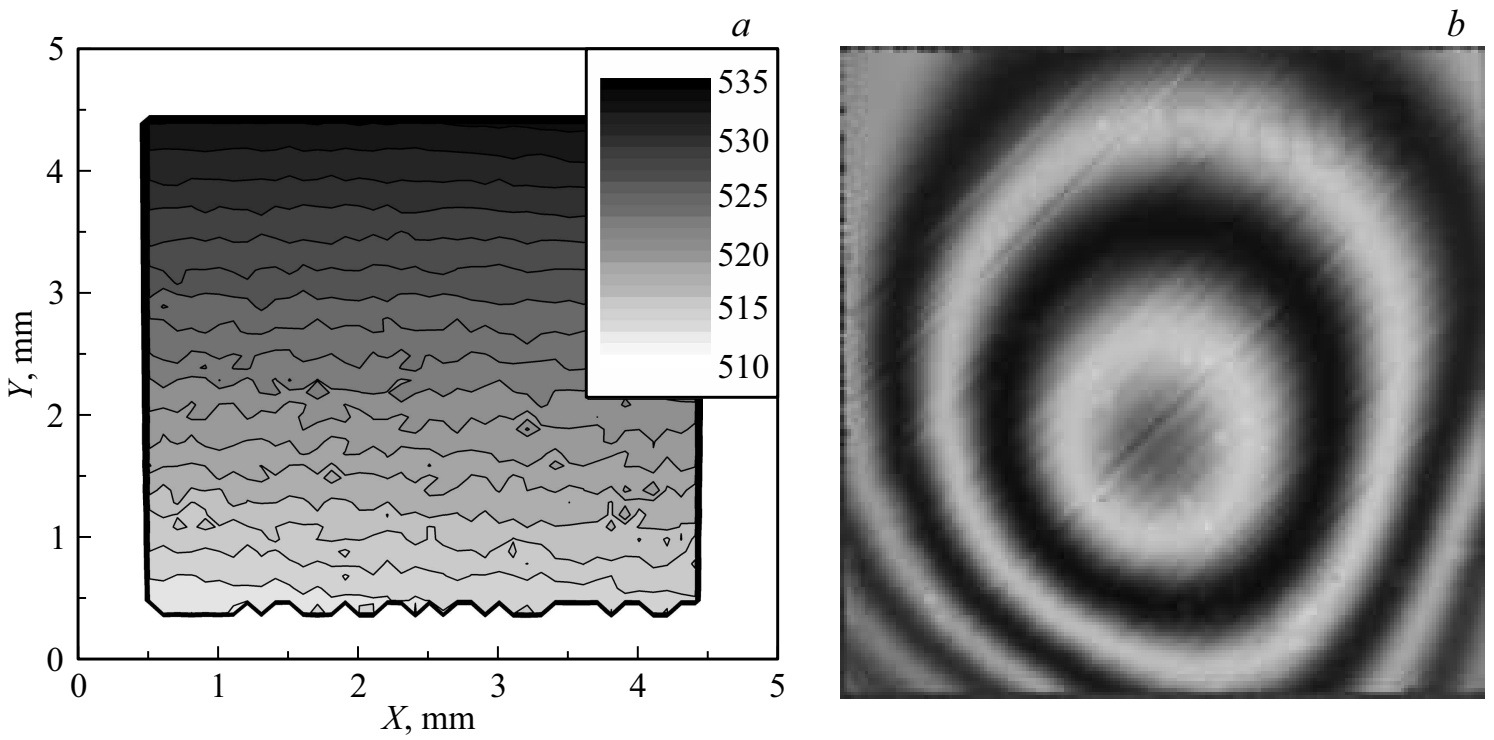

Pис. 1. $a-$ картирование толщины подложки Sub2 методом низкокогерентной оптической интерферометрии. Подложка представляет собой „клин“ с перепадом толщины до 20 мкм. На вставке указаны измеренные значения толщины подложки в микрометрах; $b$ - изображение поверхности подложки Sub3, полученное на ОИМБС Zygo.

объектом осуществлялась по оптическому волокну с фокусировкой света на образец с помощью объектива. Более подробное описание методики можно найти в работе [8].

Морфология поверхности подложек исследовалась методом оптической интерферометрии белого света (ОИМБС) на интерференционных микроскопах Talysurf CCI 2000 (поле зрения $0.9 \times 0.9 \mathrm{Mm}$ ) и Zygo New View 7300 (поле зрения - вся поверхность подложки). Также использовался метод атомно-силовой микроскопии (АСМ), микроскоп СММ-2000, размер кадра $1 \times 1$ мкм. Сочетание нескольких методов позволило охарактеризовать морфологию поверхности на разных пространственных масштабах и описать как форму, так и микрошероховатость поверхности. Рентгенодифракционные (РД) исследования выполнялись на дифрактометре Bruker D8 Discover. Проводилось картирование значений ширины кривой качания и брэгговского угла дифракционного отражения (004) алмаза по площади подложек. Подложки исследовались в 9 точках (сетка $3 \times 3$ точки с шагом 1.5 мм между точками). Съемка осуществлялась в схеме высокого разрешения с монохроматором четырехкратного отражения $\mathrm{Ge}(220)$ и коллиматором диаметром 0.3 мм на первичном пучке. Кривая качания записывалась с широкой щелью (4.5 мм) перед детектором. Брэгговский угол уточнялся с использованием кристалла анализатора $\mathrm{Ge}(220)$ трехкратного отражения. Подробности методики безэталонного измерения параметра решетки методом РД приведены в [9]. Также измерялся угол разориентации (отклонения среза) поверхности подложек относительно кристаллографической плоскости (001) по методике, описанной в [10].

\section{3. Результаты и обсуждение}

Все подложки перед началом исследований были промаркированы номерами „Sub1-Sub6“, маркировка наносилась лазером в левом верхнем углу произвольно выбранной стороны подложки. На рис. 1, $a$ приведен результат картирования толщины подложи Sub2 методом низкокогерентной оптической интерферометрии. Видно, что противоположные грани подложки непараллельны. Подложка представляет собой клин с перепадом толщины до 20 мкм, что дает величину угла между противоположными гранями $0.3^{\circ}$. По всей видимости, неплоскопараллельность граней подложки является следствием метода ее шлифовки, и эту особенность следует учитывать в технологических процессах роста и постростовой обработке структуры. Очевидно, что неплоскопараллельность граней приведет к различию углов отклонения среза для противоположных сторон подложки.

На рис. $1, b$ приведено изображение лицевой поверхности подложки Sub3, полученное на ОИМБС Zygo. На рисунке видна метка „Sub3“, нанесенная с обратной стороны. Кольцевидные интерференционные полосы не являются артефактом объектива и свидетельствуют о наличии значительного изгиба исследуемой поверхности. Также видны диагональные царапины, оставшиеся после финишной шлифовки подложки производителем. Аналогичная картина наблюдалась для обеих сторон у всех подложек в серии. Обработка результатов сканирования показала, что подложки имеют прогиб в центре с характерной величиной стрелы прогиба около 1-2 мкм. Для подложки Sub2 этот прогиб мал по сравнению с перепадом толщины подложки, по- 

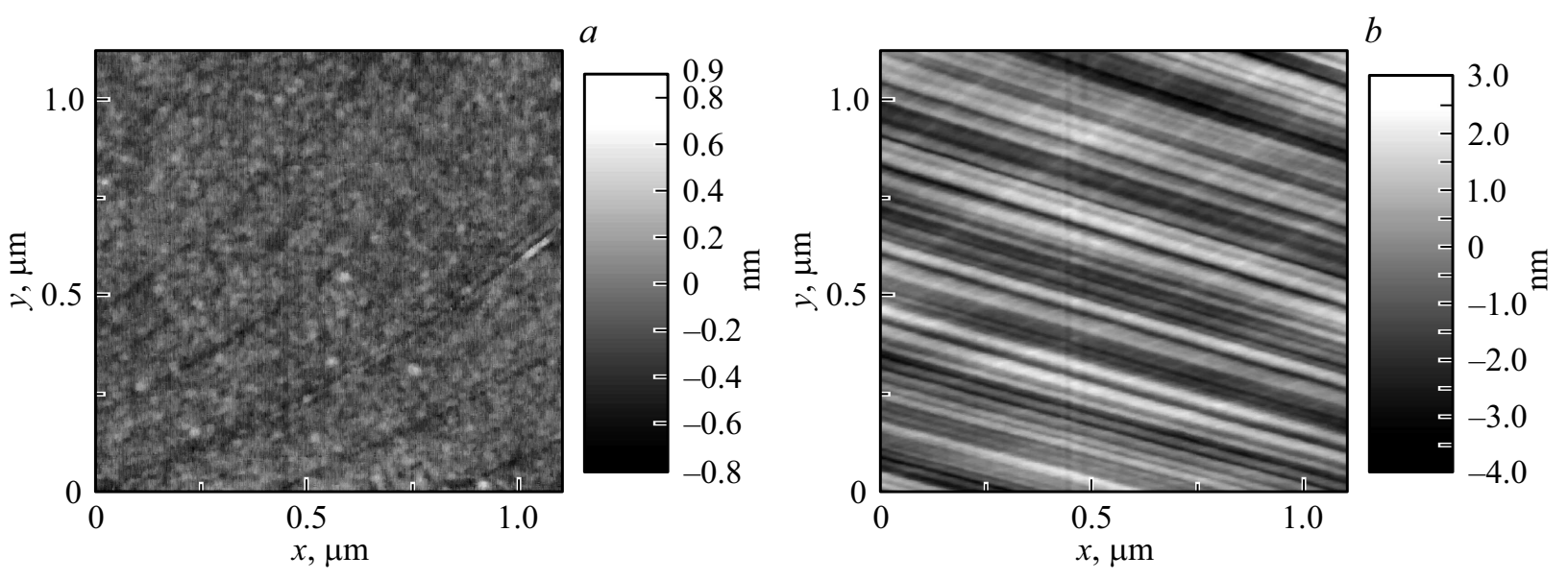

Pис. 2. АCM изображения поверхности подложек Sub1 (a) и Sub5 (b).

этому его не видно на рис. 1, $a$. Однако вычитание наклонной средней линии для результатов картирования толщины других подложек позволяет зарегистрировать прогиб в центре также и методом низкокогерентной оптической интерферометрии. Таким образом, исследованные подложки представляют собой „двояковогнутые линзы“. Соответствующие характерные значения радиуса кривизны поверхности подложек, оцененные по данным ОИМБС Zygo и Talysurf, составили от 2 до 13 метров, для подложки Sub2 кривизна поверхности заметно меньше. Данные измерения прогиба поверхности подложек на ОИМБС Zygo приведены в таблице. Там же приведены результаты измерения значений среднеквадратической шероховатости для кадра размером $1 \times 1$ мм на ОИМБС Talysurf (после вычета прогиба, аппроксимированного полиномом 2-го порядка). Полученные значения неприемлемо велики для эпитаксии и не коррелируют с величиной кривизны поверхности. Мелкомасштабная шероховатость исследовалась мето- дом АСМ на размерах кадра порядка $1 \times 1$ мкм. Характерные ACM изображения для подложек Sub1 и Sub5 приведены соответственно на рис. 2, $a$ и $b$. Было обнаружено, что по особенностям мелкомасштабной шероховатости подложки можно условно разбить на две группы. Подложки первой группы (Sub1, Sub2, Sub6) относительно гладкие, перепад высот составляет 1-2 нм (см. рис. 2,a). В отличие от них на подложках второй группы (Sub3, Sub4, Sub5) отчетливо видны следы шлифовки (рис. 2,b) глубиной 5-15нм в зависимости от образца. АСМ исследования показали, что мелкомасштабная морфология одинакова по поверхности подложки и с ее разных сторон, однако существенно отличается для разных подложек. Результаты измерения мелкомасштабной шероховатости не коррелируют с данными Zygo о кривизне поверхности и Talysurf о среднемасштабной шероховатости. Это позволяет сделать вывод о том, что при обработке поверхности подложек на формирование морфологии поверхности влияет ряд

\begin{tabular}{|c|c|c|c|c|c|c|c|c|c|c|}
\hline Sub & & $\Delta \omega$, угл.с & $S(\Delta \omega)$, угл.с & $a, \AA$ & $S(a), \AA$ & $\Delta$, град. & $R_{\text {sur }, \mathrm{M}}$ & $R_{\text {lat }}, \mathrm{M}$ & $\sigma$ АCM, нм & $\sigma$ ОИМБС, нМ \\
\hline 1 & $\begin{array}{c}\text { Лицо } \\
\text { Оборот }\end{array}$ & $\begin{array}{l}56 \\
54\end{array}$ & $\begin{array}{l}6 \\
5\end{array}$ & $\begin{array}{c}3.5667 \\
-\end{array}$ & $\begin{array}{c}3 \cdot 10^{-5} \\
-\end{array}$ & 0.8 & $\begin{array}{l}+10 \\
+7\end{array}$ & $\begin{array}{l}>100 \\
>100\end{array}$ & $\begin{array}{l}0.2 \\
0.2\end{array}$ & $\begin{array}{c}1.7 \\
-\end{array}$ \\
\hline $2 \mathrm{MC}$ & $\begin{array}{c}\text { Лицо } \\
\text { Оборот }\end{array}$ & $\begin{array}{l}48 \\
47\end{array}$ & $\begin{array}{l}3 \\
5\end{array}$ & $\begin{array}{c}3.5667 \\
-\end{array}$ & $\begin{array}{c}1 \cdot 10^{-5} \\
-\end{array}$ & 0.2 & $\begin{array}{c}- \\
+26\end{array}$ & $\begin{array}{l}>100 \\
>100\end{array}$ & $\begin{array}{c}0.2 \\
-\end{array}$ & $\begin{array}{c}5.6 \\
-\end{array}$ \\
\hline $3 \mathrm{MC}$ & $\begin{array}{c}\text { Лицо } \\
\text { Оборот }\end{array}$ & $\begin{array}{l}31 \\
31\end{array}$ & $\begin{array}{l}1 \\
2\end{array}$ & $\begin{array}{l}3.5665 \\
3.5665\end{array}$ & $\begin{array}{l}1 \cdot 10^{-5} \\
2 \cdot 10^{-5}\end{array}$ & 0.3 & $\begin{array}{l}+4 \\
+3\end{array}$ & $\begin{array}{l}>100 \\
>100\end{array}$ & $\begin{array}{l}0.9 \\
0.7\end{array}$ & $\begin{array}{c}3.4 \\
-\end{array}$ \\
\hline 4 & $\begin{array}{c}\text { Лицо } \\
\text { Оборот }\end{array}$ & $\begin{array}{l}44 \\
37\end{array}$ & $\begin{array}{l}5 \\
4\end{array}$ & $\begin{array}{l}3.5668 \\
3.5666\end{array}$ & $\begin{array}{l}1 \cdot 10^{-5} \\
4 \cdot 10^{-5}\end{array}$ & 1.4 & $\begin{array}{l}+2 \\
+3\end{array}$ & $\begin{array}{l}>100 \\
>100\end{array}$ & $\begin{array}{c}0.9 \\
-\end{array}$ & $\begin{array}{c}9.5 \\
-\end{array}$ \\
\hline $5 \mathrm{MC}$ & $\begin{array}{c}\text { Лицо } \\
\text { Оборот }\end{array}$ & $\begin{array}{l}30 \\
46\end{array}$ & $\begin{array}{l}1 \\
6\end{array}$ & $\begin{array}{l}3.5665 \\
3.5666\end{array}$ & $\begin{array}{l}2 \cdot 10^{-5} \\
2 \cdot 10^{-5}\end{array}$ & 0.1 & $\begin{array}{l}+13 \\
+4\end{array}$ & $\begin{array}{l}>100 \\
>100\end{array}$ & $\begin{array}{l}1.0 \\
-\end{array}$ & $\begin{array}{c}6.8 \\
-\end{array}$ \\
\hline 6 & $\begin{array}{c}\text { Лицо } \\
\text { Оборот }\end{array}$ & $\begin{array}{l}44 \\
64\end{array}$ & $\begin{array}{r}5 \\
13\end{array}$ & $\begin{array}{l}3.5666 \\
3.5670\end{array}$ & $\begin{array}{l}3 \cdot 10^{-5} \\
1 \cdot 10^{-5}\end{array}$ & 0.4 & $\begin{array}{l}+2 \\
+5\end{array}$ & $\begin{array}{l}>100 \\
>100\end{array}$ & $\begin{array}{c}0.2 \\
-\end{array}$ & $\begin{array}{c}4.4 \\
-\end{array}$ \\
\hline
\end{tabular}

Примечание. Параметры исследованных НРНТ подложек алмаза: средняя ширина РД кривой качания $\Delta \omega(004)$; средний параметр решетки $a$; их среднеквадратические отклонения $S$, рассчитанные по 9 точкам; отклонение среза $\Delta$; радиусы кривизны поверхности (ОИМБС) $R$ sur и решетки (РД) $R_{\text {lat }}$ и шероховатость поверхности $\sigma$ по данным АСМ и ОИМБС. Метками МС помечены моносекториальные подложки. 


\begin{tabular}{|c|c|c|}
\hline $\begin{array}{l}\Delta \omega=46^{\prime \prime} \\
a=3.5665 \AA\end{array}$ & $\begin{array}{l}\Delta \omega=45^{\prime \prime} \\
a=3.5665 \AA\end{array}$ & $\begin{array}{l}\Delta \omega=46^{\prime \prime} \\
a=3.5666 \AA\end{array}$ \\
\hline $\begin{array}{l}\Delta \omega=50^{\prime \prime} \\
a=3.5666 \AA\end{array}$ & $\begin{array}{l}\Delta \omega=49^{\prime \prime} \\
a=3.5666 \AA\end{array}$ & $\begin{array}{l}\Delta \omega=46 " \\
a=3.5666 \AA\end{array}$ \\
\hline $\begin{array}{l}\Delta \omega=39^{\prime \prime} \\
a=3.5666 \AA\end{array}$ & $\begin{array}{l}\Delta \omega=36 " \\
a=3.5666 \AA\end{array}$ & $\begin{array}{l}\Delta \omega=37^{\prime \prime} \\
a=3.5666 \AA\end{array}$ \\
\hline
\end{tabular}

Рис. 3. Схематическое изображение результатов картирования методом РД параметров подложки Sub6. Измерения проводились в 9 точках поверхности с круглым коллиматором диаметром 0.3 мм на первичном пучке, шаг сетки измерения 1.5 мм. На рисунке указаны значения РД кривой качания отражения (004) алмаза и параметра элементарной ячейки $a$.

независимых факторов на различных пространственных масштабах.

Было проведено сравнение измеренного методом ОИМБС радиуса кривизны поверхности подложек $\left(R_{\text {sur }}\right)$ с радиусом кривизны решетки алмаза, измеренным методом рентгеновской дифрактометрии $\left(R_{\text {lat }}\right)$. Измерение радиуса кривизны решетки проводилось по смещению положения $\omega$ дифракционного пика (004) алмаза при сдвиге образца относительно пятна первичного пучка. Эта стандартная методика измерения кривизны подложек, ее использование применительно к монокристаллам алмаза описано, например, в [11]. Ограничения метода, связанные с латеральными размерами подложек, не позволяют в данном случае достоверно измерять радиусы кривизны решетки больше 100 метров. Для всех подложек из исследованной партии радиус кривизны решетки оказался не меньше 100 метров, т.е. изгиб решетки пренебрежимо мал. Можно утверждать, что наблюдаемый оптическими методами „прогиб“ центральной части поверхности подложек не связан с деформацией структуры в целом, а является следствием использованной технологии шлифовки.

На рис. 3 приведены результаты картирования методом РД структурных параметров подложки Sub6. Приведены значения ширины кривой качания отражения (004) алмаза и параметра элементарной ячейки, рассчитанного по измеренному брэгговскому углу отражения. Результаты РД измерений для всех подложек также при- ведены в таблице. Величины ширины РД кривой качания $\Delta \omega$ варьируются в пределах 30-60 угл. с (без учета инструментального уширения, которое в данном случае составляет $26^{\prime \prime}$ на угле $2 \theta=119.48^{\circ}$ для $4 \times(220)$ $\mathrm{Ge}$-монохроматора). Существует разброс значений по площади подложки (среднеквадратическое отклонение $S(\Delta \omega)$, рассчитанное по 9 точкам, также приведено в таблице). Отличаются значения, измеренные с разных сторон подложки. Аналогичные различия наблюдаются и для параметра элементарной ячейки $a$. Стоит отметить, что для моносекториальных подложек были получены наименьшие значения $\Delta \omega, S(\Delta \omega)$ и $S(a)$, что говорит о лучшей однородности их структурных свойств. Различие параметра решетки $a$, как в серии, так и для одной подложки, могут обусловливаться различными концентрациями примесей как в разных процессах роста, так и за счет зонарного и секториального строения НРНТ кристалла.

В таблице также приведены измеренные значения отклонения среза подложек $\Delta$, измеренные только для одной лицевой стороны подложек. Величина отклонения варьируется в диапазоне до $1.5^{\circ}$, что характерно для НРНТ монокристаллов алмаза. Для моносекториальных подложек величина $\Delta$ меньше. По всей видимости, это обусловлено тем, что моносекториальные подложки были вырезаны из НРНТ кристаллов большего размера, точность ориентировки которых выше. В работе [12] было показано, что изменение угла разориентации подложки от 0.2 до $2^{\circ}$ может приводить к шестикратному изменению скорости травления алмаза в водородной плазме. Таким образом, величина отклонения среза является важным технологическим параметром подложки, который необходимо знать перед ее использованием для эпитаксиального роста.

\section{4. Заключение}

С точки зрения морфологии использование исследованных подложек для эпитаксии невозможно без дополнительной обработки. Не обнаружено корреляции между количественными характеристиками формы поверхности, среднемасштабной и мелкомасштабной шероховатостями. Требуется оптимизация технологии полировки подложек для достижения приемлемой морфологии. Также следует учитывать неплоскопараллельность подложек при росте и постростовой обработке эпитаксиальных структур.

Структурное качество подложек приемлемо для эпитаксиального роста алмазных пленок электронного качества, однако наблюдается различие структурных характеристик как по площади, так и с разных сторон одной подложки. При выборе рабочей стороны подложки для эпитаксиального роста желательно проводить предварительные измерения ширины рентгенодифракционных кривых качания. Также важно измерять величину отклонения среза подложки с точностью не хуже $0.2^{\circ}$. 
Очевидно, что характеризации НРНТ монокристаллов алмаза мировыми производителями только по „ювелирному“ типу недостаточно для оценки возможности их использования в качестве подложек для эпитаксиального роста CVD-алмаза. Требуется контролировать и указывать в спецификации ряд морфологических и структурных параметров, что является стандартной практикой для коммерческих подложек $\mathrm{Si}, \mathrm{A}^{\mathrm{III}} \mathrm{B}^{\mathrm{V}}$, сапфира и других материалов полупроводниковой электроники.

Работа поддержана грантом РНФ № 17-19-01580 в части подготовки подложек для эпитаксии.

\section{Список литературы}

[1] A. Tallaire, V. Mille, O. Brinza, T.N. Tran Thi, J.M. Brom, Y. Loguinov, A. Katrusha, A. Koliadin, J. Achard. Diamond Relat. Mater., 77146 (2017).

[2] R.C. Burns, A.I. Chumakov, S.H. Connell, D. Dube, H.P. Godfried, J.O. Hansen, J. Härtwig, J. Hoszowska, F. Masiello, L. Mkhonza, M. Rebak, A. Rommevaux, R. Setshedi, P. Van Vaerenbergh. J. Phys.: Condens. Matter, 21364224 364224.

[3] J. Hoszowska, A.K. Freund, E. Boller, J.P.F. Sellschop, G. Level, J. Hartwig, R.C. Burns, M. Rebak, J. Baruchel. J. Phys. D: Appl. Phys., 34, A47 (2001).

[4] Y. Kato, H. Umezawa, H. Yamaguchi, S.-i. Shikata. Diamond Relat. Mater., 29, 37 (2012).

[5] K. Tamasaku, T. Ueda, D. Miwa, T. Ishikawa. J. Phys. D: Appl. Phys., 38, A61 (2005).

[6] A.B. Muchnikov, A.L. Vikharev, J.E. Butler, V.V. Chernov, V.A. Isaev, S.A. Bogdanov, A.I. Okhapkin, P.A. Yunin, Y.N. Drozdov. Phys. Status Solidi A: Appl. Mater. Sci., 212, 2572 (2015).

[7] A.B. Muchnikov, D.B. Radishev, A.L. Vikharev, A.M. Gorbachev, A.V. Mitenkin, M.N. Drozdov, Y.N. Drozdov, P.A. Yunin. J. Cryst. Growth, 442, 62 (2016).

[8] E.V. Bushuev, V.Yu. Yurov, A.P. Bolshakov, V.G. Ralchenko, E.E. Ashkinazi, A.V. Ryabova, I.A. Antonova, P.V. Volkov, A.V. Goryunov, A.Yu. Luk'yanov. Diamond Relat. Mater., 66, 83 (2016).

[9] Ю.Н. Дроздов, П.А. Юнин. Поверхность. Рентгеновские, синхротронные и нейтронные исследования, № 1, 68 (2016).

[10] П.А. Юнин, Ю.Н. Дроздов, В.В. Чернов В.А. Исаев, С.А. Богданов, А.Б. Мучников. ФТП, 50 (12), 1647 (2016).

[11] X. Xie, X. Wang, Y. Peng, Y. Cui, X. Chen, X. Hu, X. Xu, P. Yu, R. Wang. J. Mater. Sci. Mater. Electron., 28, 9813 (2017).

[12] O.A. Ivanov, A.B. Muchnikov, V.V. Chernov, S.A. Bogdanov, A.L. Vikharev, J.E. Butler. Mater. Lett., 151, 115 (2015).

Редактор Г.А. Оганесян

\section{Investigation of the structural and morphological properties of HPHT diamond substrates}

P.A. Yunin ${ }^{1}$, P.V. Volkov ${ }^{1}$, Yu.N. Drozdov' ${ }^{1}$, A.V. Koliadin ${ }^{3}$, S.A. Korolev ${ }^{1}$, D.B. Radischev ${ }^{2}$, E.A. Surovegina ${ }^{1}$, V.I. Shashkin ${ }^{1}$

${ }^{1}$ Institute for Physics of Microstructures, Russian Academy of Sciences, 603087 Nizhny Novgorod, Russia 2 Institute of Applied Physics, Russian Academy of Sciences, 603950 Nizhny Novgorod, Russia 3 LLC „New Diamond Technology“, 197706 St. Petersburg, Russia

Abstract By methods of white light interference microscopy, atomic force microscopy and $X$-ray diffractometry, complex studies of the morphological and structural properties for a series of single crystal HPHT diamond substrates have been carried out. Techniques are described that make it possible to characterize the most critical for epitaxial application parameters of diamond substrates using laboratory equipment. It is shown that the characterization of diamond substrates only in terms of the jewelry type is not enough for assessing the possibility of their use for the epitaxial growth of CVD diamond. 